\title{
Conversion of Existing Calorimetrically Determined Thermodynamic Properties to the Basis of the International Practical Temperature Scale of 1968
}

\author{
Thomas B. Douglas \\ Institute for Basic Standards, National Bureau of Standards, Washington, D.C. 20234
}

(June 20, 1969)

\begin{abstract}
Formulas are derived for converting the relative enthalpy, heat capacity, entropy, and Gibbs energy from the basis of one practical temperature scale to the basis of another, when these properties on either scale have been derived from calorimetric measurements of enthalpy as though that scale were the thermodynamic one. These formulas are directly applicable for converting certain other properties as well. The conversion relates the values of the property at the same numerical temperature on both scales. The formulas, given as exact infinite series, are applicable to widely differing scales, one of which may vary linearly with a temperature-measuring quantity such as electrical resistance. However, great simplification is well within most calorimetric accuracy when the conversion is from the International Practical Temperature Scale of 1948 to the corresponding scale of 1968, which has recently replaced it, provided the heat capacity is not changing abnormally rapidly, as in a transition region. For convenient application to conversion between these two scales, relatively simple numerical equations are derived giving the differences between the two scales at temperatures from $90 \mathrm{~K}$ to $10,000 \mathrm{~K}$. The problem of avoiding the introduction of discontinuities with temperature in converted tables, arising from the existing discontinuities in the temperature derivative of the differences between the two scales, is discussed.
\end{abstract}

Key words: Existing property tables; IPTS-48; IPTS-68; practical-scale differences; temperaturescale conversion; thermodynamic properties.

\section{Introduction}

The International Practical Temperature Scale of 1968 (“IPTS-68”) was adopted in 1968 $[1]^{1}$ as a replacement for the corresponding scale of 1948 (amended edition of 1960) ("IPTS-48"). The IPTS-68 serves the same general purposes as the IPTS-48, but better: its specification is more consistent with current experimental capabilities for reproducing temperature, it more closely approximates the thermodynamic teinperature scale, and it extends the temperature range downward (from approximately $90 \mathrm{~K}$ in the IPTS-48 to approximately $14 \mathrm{~K}$ in the IPTS-68).

The great preponderance of quantitative macroscopic properties of matter vary appreciably with temperature. As a consequence, it will be of interest and concern as to the extent to which numerous existing values of these properties, expressed in reference to temperatures on the IPTS-48 or an earlier scale, would change if referred to temperatures on the IPTS-68. In the case of the most accurate values it will be desirable to make the actual conversion - for the sake of future consistency, for facilitating direct comparison with future values expressed in reference to the IPTS-68, and for better thermodynamic accuracy when this is significant. In what will undoubtedly be the more common case of less accurate values, one will want to know the magnitudes of the errors neglected if such a conversion is not made. Incidentally, tables of thermodynamic properties of ideal gases derived statistical-mechanically from molecular-constant data

\footnotetext{
${ }^{1}$ Figures in brackets indicate the literature references at the end of this paper.
} 
are based on the thermodynamic temperature scale (as well as on the values of the physical constants used), and are hence independent of any practical temperature scale.

This article is limited to a consideration of the more common thermodynamic properties derived from calorimetric data-enthalpy, heat capacity, entropy, and Gibbs (free) energy-with particular attention to the substitution of the IPTS-68 for the IPTS-48 as a temperature basis. The differences between these two scales (listed numerically in table 4 of this article) are not so large but that very simple procedures which have been previously used, ${ }^{2}$ and which are virtually self-evident, are adequate within the accuracy of most existing calorimetric data. However, it seems worthwhile to outline systematically the rigorous conversion procedures, which may be required not only in regions of sharp transitions but also in those cases where the correction terms are large, as when one is converting from an arbitrary temperature scale such as one related very simply (for convenience) to thermometer resistance, thermocouple electromotive force, or radiative brightness.

In section 2, conversion formulas (exact as infinite series) are derived for converting the thermodynamic properties named above to the basis of a new temperature scale, in terms of the difference between the two scales as a continuous function of temperature. The thermodynamic properties are assumed to be based on experimental measurements of enthalpy increments that span the entire temperature range of interest. Thus the correction to be applied to an enthalpy increment involves merely associating it with different boundary temperatures, but the corrections to the properties derived from the enthalpy (heat capacity, entropy, etc.) involve in addition what is essentially a repetition of the differentiation or integration as if the new temperature scale, not the old, corresponds to true thermodynamic temperatures. The formulas derived convert the properties at a given temperature on the old scale to those at the same numerical value of temperature on the new scale, thus preserving the "round" temperature values of most tables. When, instead, it is desired to make conversion to a fixed-point temperature whose values are different on the two scales, the additional step simply involves the variation of the given property with temperature on one of the scales, and can be readily carried out by standard thermodynamic procedures.

Some non-calorimetric properties may be converted to the basis of the new temperature scale using the same formulas derived in this article. For example, if the linear coefficient of thermal expansion is defined as $(1 / l)(d l / d t)$, then this coefficient or $\ln l$ can replace $C_{p}$ or $H$, respectively.

In section 2.5 it is shown what modifications are needed when extrapolation of calorimetric data to $0 \mathrm{~K}$ has been effected by such means as the well-known " $T^{3}$ " law. Simple approximate correction formulas, obtained by dropping all terms of the exact infinite series that are of no practical value when the conversion is from the IPTS-48 to the IPTS-68 and outside sharptransition regions, are given in section 2.6.

In section 3, a numerical equation giving the difference between the IPTS- 68 and the IPTS-48 $\left(T_{68}-T_{48}\right.$, or $\left.t_{68}-t_{48}\right)$ as a function of temperature is derived for each of the four temperature ranges above the oxygen boiling point (approximately $90 \mathrm{~K}$ ) that are defined separately by the IPTS-68. Values of $T_{68}-T_{48}$ and its first temperature derivative, calculated from these equations, are tabulated up to $10,000 \mathrm{~K}$ in section 3.6 , for use in the formulas of section 2 ; the values of $T_{68}-T_{48}\left(=t_{68}-t_{48}\right)$ agree perfectly with a recently published tabulation [1]. Though the IPTS-68 is defined from 13.81 to $90.188 \mathrm{~K}$, the IPTS -48 was not, so the two scales cannot be compared in this temperature range. (Tentative differences in this range between the IPTS-68 and each of four "national" scales are being published, but it is stated that these differences may undergo changes of up to several millikelvins when the national scales are finally compared accurately to the IPTS-68 [3].)

The first temperature derivative of $T_{68}-T_{48}$ is continuous at the ice point $\left(0{ }^{\circ} \mathrm{C}\right)$, but has sharp discontinuities at the "antimony" and gold points (approximately 631 and $1064{ }^{\circ} \mathrm{C}$ ) which will

${ }^{2}$ E.g., several years ago the author derived corrections to convert the thermodynamic properties of mercury from the basis of practical temperature scales to what was at that time regarded as the best available approximation to the scale of thermodynamic temperatures [2]. 
introduce corresponding small discontinuities at these temperatures into a heat capacity-temperature function that has previously been smoothed. This problem is discussed in section 3.5. where suggested "smoothing" equations to eliminate these discontinuities are derived.

\section{Derivation of General Conversion Formulas}

\subsection{Enthalpy $(H)$}

The conversion formula derived in this section for enthalpy (and those derived in sections 2.2-2.6 for other properties as well) are for clarity expressed specifically in terms of conversion from the IPTS-48 to the IPTS-68. Because these formulas (in sections 2.1-2.5) are exact, any other pair of continuous temperature scales may be used instead. For example, one of these scales might be defined as some linear function of thermometer resistance or thermocouple electromotive force.

The formulation is given in terms of what is now called the International Practical Kelvin (absolute) temperature $(T)$, which is numerically greater than the International Practical Celsius temperature $(t)$ by 273.15. This constant was adopted for the IPTS-68 [1], and for the IPTS-48 in 1954. (Between 1948 and 1954 this constant was not officially defined, but was usually taken as 273.16.) Thus we shall always use

$$
T_{68}=t_{68}+273.15
$$

and

$$
T_{48}=t_{48}+273.15
$$

designating the value of the temperature on either IPTS as Kelvin or Celsius interchangeably as may prove convenient.

We shall often find it convenient to refer specifically to a pair of temperatures ${ }^{3}$ (referred to as temperatures 1 and 2 and symbolized by single prime and double prime, respectively), and consider the case of converting the enthalpy (or any other property) from its value on the basis of the IPTS-48 at temperature 1 to its value on the basis of the IPTS-68 at temperature 2. As stated in the introduction (sec. 1), these two temperatures will be related to each other such that numerically

$$
T_{68}^{\prime \prime}=T_{48}^{\prime}
$$

Since such thermodynamic properties as enthalpy cannot be assigned absolute values and therefore are usually expressed numerically as the magnitude in excess of the enthalpy at a reference temperature, it will be convenient to use the symbol $H$ to represent this relative enthalpy. Because it simplifies the treatment, the reference temperature is to be restricted to one having the same value on both temperature scales (e.g., $273.16 \mathrm{~K}$, but not $298.15 \mathrm{~K}$ ). The situation under discussion may be clarified somewhat by reference to figures 1 and 2 , which are schematic and are not to be taken as implying a given sign or magnitude of $\left(T_{68}^{\prime}-T_{48}^{\prime}\right)$ or $\left(T_{68}^{\prime \prime}-T_{48}^{\prime \prime}\right)$.

The relative enthalpy will be assumed to be given by heat measurements at constant pressure, but it will be convenient to omit the indication of the constancy of pressure from derivatives of enthalpy. The lack of both a single and a double prime will imply a temperature unspecified in relation to temperatures 1 and 2 . For convenience we shall abbreviate according to the further definitions:

$$
\begin{gathered}
C_{P}^{68} \equiv d H / d T_{68} ; \\
C_{P}^{48} \equiv d H / d T_{48} ; \\
\mu\left[=\mu\left(T_{68}\right)\right] \equiv T_{68}-T_{48} \text { at a given temperature. }
\end{gathered}
$$

${ }^{3}$ Subsequent use of the word "temperature" instead of "temperature value" is restricted to mean "hotness." 


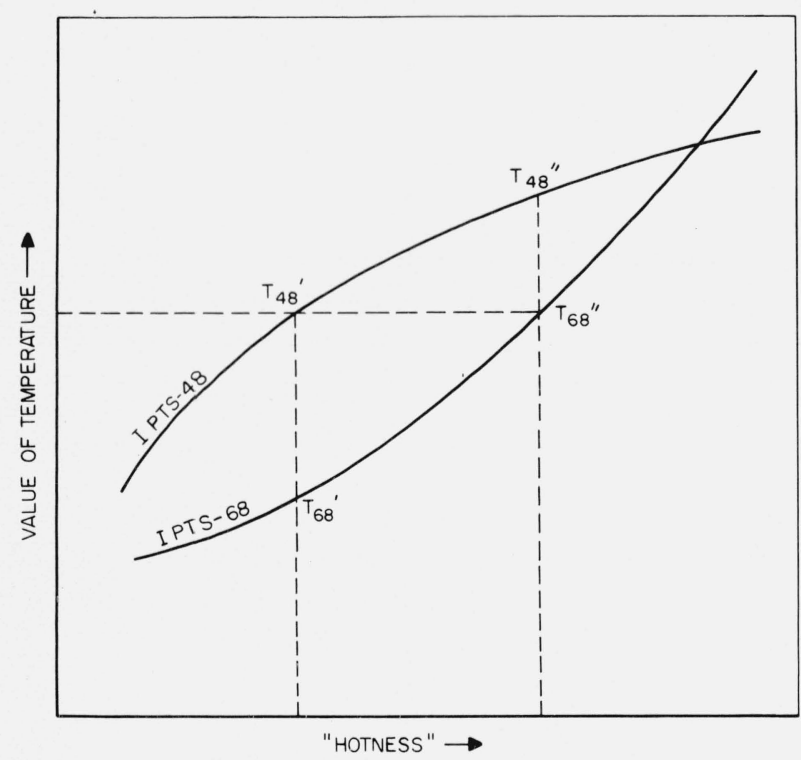

FIGURE 1. Schematic example illustrating the relation of $\mathrm{T}_{48}$ and $\mathrm{T}_{68}$ to "hotness."

(The thermodynamic or any other temperature scale whose temperature values continually increase with increasing "hotness" may be used as a quantitative measur of "hotness.")

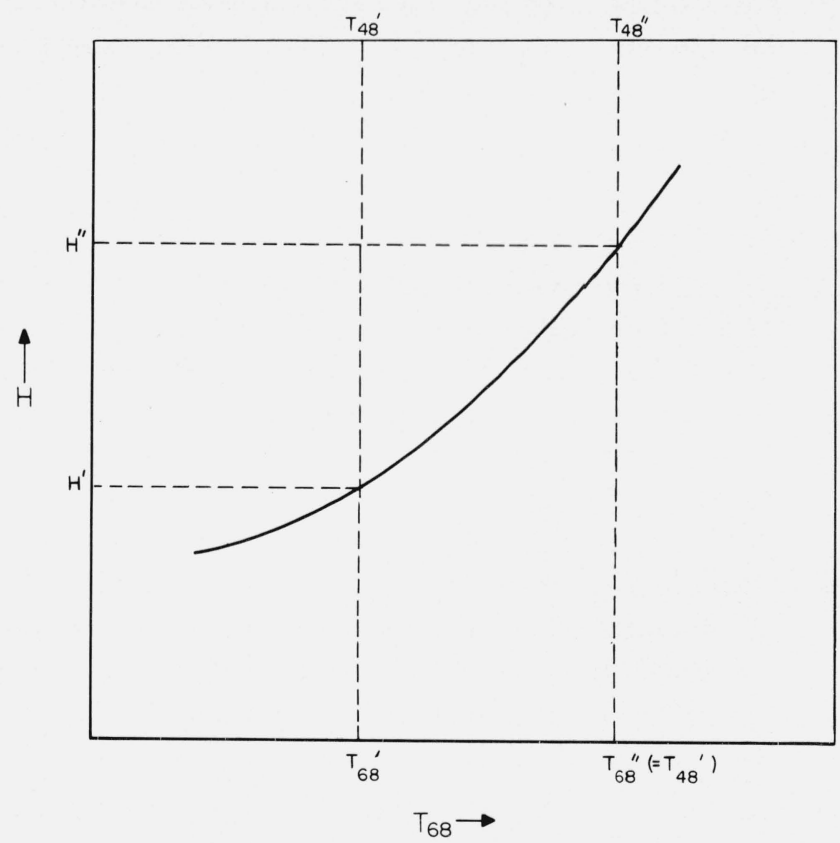

FIGURE 2. Schematic example illustrating the conventions used in converting relative enthalpy $(\mathrm{H})$ from the basis of one temperature scale (e.g., $\mathrm{T}_{48}$ ) to that of another scale (e.g., $\left.\mathrm{T}_{68}\right)$.

$H^{\prime}$ (at $T_{48}^{\prime}$ ) is converted to $H^{\prime \prime}$ (at $T_{68}^{\prime \prime}$ ).

In more specific form for future reference, eq (6) can of course be written

$$
\mu^{\prime}=T_{68}^{\prime}-T_{48}^{\prime}
$$

or

$$
\mu^{\prime \prime}=T_{68}^{\prime \prime}-T_{48}^{\prime \prime}
$$

From eq (3) and (8),

$$
\mu^{\prime \prime}=T_{48}^{\prime}-T_{48}^{\prime \prime}
$$

From eq (6),

$$
\frac{d T_{48}}{d T_{68}}=1-\frac{d \mu}{d T_{68}}
$$

and therefore

$$
\frac{d T_{68}}{d T_{48}}=\frac{1}{1-\frac{d \mu}{d T_{68}}}
$$

The Taylor expansion of $H\left(T_{48}\right)$ about $H^{\prime}$ is [remembering eq (5)]

$$
H=H^{\prime}+\left(T_{48}-T_{48}^{\prime}\right)\left(C_{P}^{48}\right)^{\prime}+\frac{1}{2}\left(T_{48}-T_{48}^{\prime}\right)^{2}\left(\frac{d C_{P}^{48}}{d T_{48}}\right)^{\prime}+\frac{1}{6}\left(T_{48}-T_{48}^{\prime}\right)^{3}\left(\frac{d^{2} C_{P}^{48}}{d T_{48}^{2}}\right)^{\prime}+\ldots
$$

Substituting $H^{\prime \prime}$ for $H$ and $T_{48}^{\prime \prime}$ for $T_{48}$ in eq (12), then replacing $\left(T_{48}^{\prime \prime}-T_{48}^{\prime}\right)$ from eq (9), gives the desired enthalpy correction $\delta H$, 


$$
\delta H=H^{\prime \prime}-H^{\prime}=-\mu^{\prime \prime}\left(C P_{p}^{8}\right)^{\prime}+\frac{1}{2}\left(\mu^{\prime \prime}\right)^{2}\left(\frac{d C_{p}^{8}}{d T_{48}}\right)^{\prime}-\frac{1}{6}\left(\mu^{\prime \prime}\right)^{3}\left(\frac{d^{2} C_{P}^{48}}{d T_{48}^{2}}\right)^{\prime}+\ldots
$$

Equation (13) is in a convenient form: $H$ and its derivatives with respect to $T_{48}$ are presumed known (or evaluable) at the round value of temperature $T_{48}=T_{48}^{\prime}$, whereas $\mu$ is assumed to be known as a function of $T_{68}$ and so can readily be evaluated at the temperature where $T_{68}=T_{68}^{\prime \prime}$ $\left(=T_{48}^{\prime}\right)$, also a round value of temperature.

Equation (13) is exact (as an infinite series). However, of the rhs (right-hand side), only the first term is, usually, not negligible in practice (see sec. 2.6).

Reverting temporarily to the usual definition of the symbol $H$ as representing absolute enthalpy at some one temperature, let us exemplify enthalpy relative to that at a temperature where $\mu \neq 0$ by $H-H_{298.15}$. Obviously $\delta\left(H-H_{298.15}\right) \equiv \delta\left(H-H_{c}\right)-\delta\left(H_{298.15}-H_{c}\right)$. Each of the two terms of the right-hand side be evaluated separately from eq (13) with reference to a temperature $c$ (say, $0 \mathrm{~K}$ ) where $\mu=0$.

\subsection{Heat Capacity $\left(C_{p}\right)$}

The Taylor expansion of heat capacity as a function of $T_{48}$ about $\left(C_{p}^{48}\right)^{\prime}$ is [after replacing $C_{P}^{48}$ by $\left(C_{P}^{48}\right)^{\prime \prime}, T_{48}$ by $T_{48}^{\prime \prime}$, and then $\left(T_{48}^{\prime \prime}-T_{48}^{\prime}\right)$ from eq $\left.(9)\right]$

$$
\left(C_{P}^{48}\right)^{\prime \prime}=\left(C_{P}^{48}\right)^{\prime}-\mu^{\prime \prime}\left(\frac{d C_{p}^{48}}{d T_{48}}\right)^{\prime}+\frac{1}{2}\left(\mu^{\prime \prime}\right)^{2}\left(\frac{d^{2} C_{7}^{48}}{d T_{48}^{2}}\right)^{\prime}-\ldots
$$

From eq (4), (5), and (11),

$$
\left(C_{p}^{8}\right)^{\prime \prime}=\left(C_{p}^{68}\right)^{\prime \prime}\left[\frac{1}{1-\left(\frac{d \mu}{d T_{68}}\right)^{\prime \prime}}\right] .
$$

Substituting for $\left(C_{P}^{48}\right)^{\prime \prime}$ from eq (15) into eq (14) and then solving for $\left(C_{P}^{68}\right)^{\prime \prime}-\left(C_{P}^{48}\right)^{\prime}$ gives the desired heat-capacity correction $\delta C_{P}$,

$\delta C_{P}=\left(C_{P}^{68}\right)^{\prime \prime}-\left(C_{P}^{48}\right)^{\prime}=-\left(\frac{d \mu}{d T_{68}}\right)^{\prime \prime}\left(C_{P}^{48}\right)^{\prime}+\left[1-\left(\frac{d \mu}{d T_{68}}\right)^{\prime \prime}\right]\left[-\mu^{\prime \prime}\left(\frac{d C_{P}^{48}}{d T_{48}}\right)^{\prime}+\frac{1}{2}\left(\mu^{\prime \prime}\right)^{2}\left(\frac{d^{2} C_{P}^{48}}{d T_{48}^{2}}\right)^{\prime}-\ldots .\right.$.

Equation (16) is exact (as an infinite series). In practice, $\left|\frac{d \mu}{d T_{68}}\right| \ll 1$, so that the factor $\left[1-\left(\frac{d \mu}{d T_{68}}\right)^{\prime \prime}\right]$ may be replaced by 1 (approximate).

\subsection{Absolute "Third-Law" Entropy (S)}

We wish to find the equivalent of $\left(S^{68}\right)^{\prime \prime}-\left(S^{48}\right)^{\prime}$, each term of which is taken to represent entropy relative to that at $0 \mathrm{~K}$ (absolute entropy if the Third Law of Thermodynamics holds) and is . evaluated from the enthalpy data at its respective indicated temperature value on the assumption that the respective indicated IPTS represents the thermodynamic temperature.

Since both $\left(S^{68}\right)^{\prime \prime}$ and $\left(S^{48}\right)^{\prime}$ involve integration down to $0 \mathrm{~K}$, it is convenient to formulate them separately, then take the difference. Consider $d S^{68}$ and $d S^{48}$ over a definite infinitesimal temperature range, $d H$ being a fixed experimentally determined quantity. Then

$$
d S^{68}=\frac{d H}{T_{68}}
$$

and

$$
d S^{48}=\frac{d H}{T_{48}} .
$$


Replacing $d H$ from eq (5) and $T_{68}$ from eq (6), eq (17) becomes

$$
d S^{68}=\left(\frac{C_{P}^{48}}{T_{48}+\mu}\right) d T^{48}
$$

Integrating eq (19),

$$
\left(S^{68}\right)^{\prime \prime}=\int_{0}^{T_{48}^{\prime}}\left(\frac{C 4_{8}^{8}}{T_{48}+\mu}\right) d T_{48}+\left(S^{68}\right)^{\prime \prime}-\left(S^{68}\right)^{\prime}
$$

Similarly using eq (5), the integration of eq (18) gives

$$
\left(S^{48}\right)^{\prime}=\int_{0}^{T_{48}^{\prime 8}} \frac{C_{P}^{48}}{T_{48}} d T_{48}
$$

Subtracting eq (21) from eq (20),

$$
\left(S^{68}\right)^{\prime \prime}-\left(S^{48}\right)^{\prime}=-\int_{0}^{T_{48}^{\prime}} \frac{\mu C_{7^{8}} d T_{48}}{T_{48}\left(T_{48}+\mu\right)}+\left(S^{68}\right)^{\prime \prime}-\left(S^{68}\right)^{\prime}
$$

$\left(S^{68}\right)^{\prime \prime}-\left(S^{48}\right)^{\prime}$ is the desired correction $(\equiv \delta S)$, but it remains (a) to formulate $\mu\left(T_{48}\right)$ from the presumably known $\mu\left(T_{68}\right)$ for use in eq $(22)$, and (b) to substitute for $\left(S^{68}\right)^{\prime \prime}-\left(S^{68}\right)^{\prime}$ in terms of known quantities, remembering that $S^{48}$ and its derivatives with respect to $T_{48}$ are known at $\mathrm{T}_{48}=T_{48}^{\prime}$, whereas $\mu$ and its derivatives with respect to $T_{68}$ are known at $T_{68}=T_{68}^{\prime \prime}$, numerically the same value of temperature.

Expanding $\mu\left(T_{48}\right)$ about $\mu^{\prime \prime}$, then setting $\mu=\mu^{\prime}$,

$$
\mu^{\prime}=\mu^{\prime \prime}+\left(T_{48}^{\prime}-T_{48}^{\prime \prime}\right)\left(\frac{d \mu}{d T_{48}}\right)^{\prime \prime}+\frac{1}{2}\left(T_{48}^{\prime}-T_{48}^{\prime \prime}\right)^{2}\left(\frac{d^{2} \mu}{d T_{48}^{2}}\right)^{\prime \prime}+\ldots
$$

Substituting from eq (9), eq (23) gives

$$
\mu^{\prime}=\mu^{\prime \prime}\left[1+\left(\frac{d \mu}{d T_{48}}\right)^{\prime \prime}\right]+\frac{1}{2}\left(\mu^{\prime \prime}\right)^{2}\left(\frac{d^{2} \mu}{d T_{48}^{2}}\right)^{\prime \prime}+\ldots .
$$

Using eq (11), we can write

$$
\frac{d \mu}{d T_{48}} \equiv \frac{d \mu}{d T_{68}} \frac{d T_{68}}{d T_{48}}=\frac{\frac{d \mu}{d T_{68}}}{1-\frac{d \mu}{d T_{68}}}
$$

Differentiating eq (25) with respect to $T_{48}$ gives

$$
\frac{d^{2} \mu}{d T_{48}^{2}}=\frac{\frac{d^{2} \mu}{d T_{68}^{2}}}{\left(1-\frac{d \mu}{d T_{68}}\right)^{3}} .
$$

Substituting from eq (25) and (26) for the derivatives in eq (24) gives

$$
\mu^{\prime}=\frac{\mu^{\prime \prime}}{1-\left(\frac{d \mu}{d T_{68}}\right)^{\prime \prime}}+\frac{\frac{1}{2}\left(\frac{d^{2} \mu}{d T_{68}^{2}}\right)^{\prime \prime}\left(\mu^{\prime \prime}\right)^{2}}{\left[1-\left(\frac{d \mu}{d T_{68}}\right)^{\prime \prime}\right]^{3}}+\ldots
$$


We are assuming $\mu$ to be available in the form $\mu\left(T_{68}\right)$. Consider any given value of $T_{48}$ in the integrand of eq (22); if we temporarily call it $T_{48}^{\prime}$ (not to be confused with the $T_{48}^{\prime}$ which forms the upper limit of integration), then the $\mu$ to be associated with it is, of course, $\mu$ at $T_{48}=T_{48}^{\prime}$, or $\mu^{\prime}$. This $\mu^{\prime}$ can be obtained from eq (27), since, in view of eq (3), the corresponding $\mu^{\prime \prime}$ is $\mu$ at $T_{68}=T_{68}^{\prime \prime}=T_{48}^{\prime}$. Hence the $\mu$ to use in the integrand of eq (22) is $\mu^{\prime}$ of eq (27), where $\mu^{\prime \prime}$ and its derivatives with respect to $T_{68}$ are evaluated, with $T_{68}$ then replaced by $T_{48}$. Expressed more concisely, where $\psi$ designates a definite function defined by the rhs of eq (27):

$$
\left.\begin{array}{c}
\mu^{\prime}=\psi\left(T_{68}^{\prime \prime}\right) \\
\mu\left(T_{48}\right)=\psi\left(T_{48}\right)
\end{array}\right\}
$$

Although eq (27) as an infinite series is exact, in practice $\left|\frac{d \mu}{d T_{68}}\right| \ll 1, \mu\left|\frac{d^{2} \mu}{d T_{68}^{2}}\right| \ll 1$, etc., so that

$$
\mu^{\prime} \simeq \mu^{\prime \prime}
$$

Turning to the formulation of $\left(S^{68}\right)^{\prime \prime}-\left(S^{68}\right)^{\prime}$ [needed in using eq (22)], let us expand $S^{68}\left(T_{48}\right)$ about $\left(S^{68}\right)^{\prime}$. Then successively replacing $S^{68}$ by $\left(S^{68}\right)^{\prime \prime}, T_{48}$ by $T_{48}^{\prime \prime}$, and $T_{48}^{\prime \prime}-T_{48}^{\prime}$ from eq (9), we get

$$
\left(S^{68}\right)^{\prime \prime}-\left(S^{68}\right)^{\prime}=-\mu^{\prime \prime}\left(\frac{d S^{68}}{d T_{48}}\right)^{\prime}+\frac{1}{2}\left(\mu^{\prime \prime}\right)^{2}\left(\frac{d^{2} S^{68}}{d T_{48}^{2}}\right)^{\prime}-\ldots
$$

From eq (19)

$$
\frac{d S^{68}}{d T_{48}}=\frac{C{ }^{8}}{T_{48}+\mu},
$$

and differentiating eq (31),

$$
\frac{d^{2} S^{68}}{d T_{48}^{2}}=\left(\frac{1}{T_{48}+\mu}\right) \frac{d C_{P}^{48}}{d T_{48}}-\frac{C_{P}^{48}}{\left(T_{48}+\mu\right)^{2}}\left(1+\frac{d \mu}{d T_{48}}\right)
$$

From eq (25),

$$
1+\frac{d \mu}{d T_{48}}=\frac{1}{1-\frac{d \mu}{d T_{68}}} .
$$

Equations (31)-(33) convert eq (30) into

$$
\left(S^{68}\right)^{\prime \prime}-\left(S^{68}\right)^{\prime}=-\frac{\mu^{\prime \prime}\left(C p^{8}\right)^{\prime}}{T_{48}^{\prime}+\mu^{\prime}}\left\{1+\frac{\frac{1}{2} \mu^{\prime \prime}}{\left[T_{48}^{\prime}+\mu^{\prime}\right]\left[1-\left(\frac{d \mu}{d T_{68}}\right)^{\prime}\right]}\right\}+\frac{\frac{1}{2}\left(\mu^{\prime \prime}\right)^{2}}{T_{48}^{\prime}+\mu^{\prime}}\left(\frac{d C_{P}^{48}}{d T_{48}}\right)^{\prime}+\ldots
$$

Finally, substituting for $\left(S^{68}\right)^{\prime \prime}-\left(S^{68}\right)^{\prime}$ into eq (22) from eq (34) gives the desired entropy correction $\delta S$,

$$
\begin{array}{r}
\delta S=\left(S^{68}\right)^{\prime \prime}-\left(S^{48}\right)^{\prime}=-\int_{0}^{T_{48}^{\prime}} \frac{\mu C_{78}^{48} d T_{48}}{T_{48}\left(T_{48}+\mu\right)}-\frac{\mu^{\prime \prime}\left(C_{P}^{48}\right)^{\prime}}{T_{48}^{\prime}+\mu^{\prime}}\left\{1+\frac{\frac{1}{2} \mu^{\prime \prime}}{\left.\left[T_{48}^{\prime}+\mu^{\prime}\right]\left[1-\left(\frac{d \mu}{d T_{68}}\right)^{\prime}\right]\right\}}\right. \\
+\frac{\frac{1}{2}\left(\mu^{\prime \prime}\right)^{2}}{T_{48}^{\prime}+\mu^{\prime}}\left(\frac{d C_{P^{8}}^{8}}{d T_{48}}\right)^{\prime}+\ldots
\end{array}
$$

where, if eq (29) is not a sufficiently good approximation, $\mu\left(T_{48}\right)$ in the integrand can be evaluated as $\mu^{\prime}$ from eq (27) [in view of eq (28)], and where $\mu^{\prime}$ and $\left(\frac{d \mu}{d T_{68}}\right)^{\prime}$ can also be found from eq (27). 
The contribution of any first-order process (such as a heat of transition, fusion, or vaporization) to $C{ }^{8} 8 T_{48}$ in eq (35) is indeterminate, and must be replaced by $d H$, whose integral is the heat of that process.

Equation (35) as an infinite series is exact, but it does assume calorimetric evaluation of $C \$^{8}\left(T_{48}\right)$ all the way down to $0 \mathrm{~K}$. When this ascuristion is invalid, see section 2.5 .

\subsection{Other Thermodynamic Functions}

The other thermodynamic functions commonly used in chemical thermodynamics can be readily converted from the basis of one temperature scale to that of another using the corrections found above (secs. 2.1 and 2.3) for the enthalpy, $H$, and the absolute entropy, $S$. For example, consider the enthalpy function $H / T$, more explicitly written $\left(H_{T}-H_{0}\right) / T$ when $H$ is understood to represent the enthalpy at temperature $T$ relative to the enthalpy at absolute zero. Using eq (3) and our previous notation, the correction $\delta(H / T)$ is given by

$$
\delta\left(\frac{H}{T}\right)=\frac{H^{\prime \prime}}{T_{68}^{\prime \prime}}-\frac{H^{\prime}}{T_{48}^{\prime}}=\frac{H^{\prime \prime}-H^{\prime}}{T_{48}^{\prime}}
$$

or simply the correction to the enthalpy, divided by the value of the Kelvin temperature. The correction $\delta G$ to the Gibbs energy is given by

$$
\delta G=\left(G^{68}\right)^{\prime \prime}-\left(G^{48}\right)^{\prime}=\left[H^{\prime \prime}-T_{68}^{\prime \prime}\left(S^{68}\right)^{\prime \prime}\right]-\left[H^{\prime}-T_{48}^{\prime}\left(S^{48}\right)^{\prime}\right]=H^{\prime \prime}-H^{\prime}-T_{48}^{\prime}\left[\left(S^{68}\right)^{\prime \prime}-\left(S^{48}\right)^{\prime}\right],
$$

where $H$ and $G$ are both understood to be relative to the enthalpy $H$ at some one temperature which has the same value on both temperature scales. The correction $\delta(-G / T)$ to the "free-energy function" $-\left(G_{T}-H_{0}\right) / T$, when the latter is abbreviated to $-G / T$, is analogous to eq (36):

$$
\delta\left(-\frac{G}{T}\right)=\left[-\frac{\left(G^{68}\right)^{\prime \prime}}{T_{68}^{\prime \prime}}\right]-\left[-\frac{\left(G^{48}\right)^{\prime}}{T_{48}^{\prime}}\right]=-\frac{\left[\left(G^{68}\right)^{\prime \prime}-\left(G^{48}\right)^{\prime}\right]}{T_{48}^{\prime}},
$$

where $-\left[\left(G^{68}\right)^{\prime \prime}-\left(G^{48}\right)^{\prime}\right]$ is given by eq $(37)$.

\subsection{Modifications of Conversion Procedure when Significant Extrapolation to $0 \mathrm{~K}$ was Made Using a Theoretical Relation}

In evaluating thermodynamic functions based on $0 \mathrm{~K}$, the heat capacity is measured to as low a temperature as practical and then extrapolation to $0 \mathrm{~K}$ is carried out on some more or less simple theoretical basis. The following treatment of the conversion to the basis of a new temperature scale will be exemplified by the use of the simple " $T^{3}$ " law. If the extrapolation has been from such a low temperature that the heat capacity at all lower temperatures is very small, the modifications are of little importance. However, a great many heat-capacity investigations have involved experimental measurements only down to the triple point of nitrogen (or oxygen), and it is desirable to know whether the required modifications are significant in preserving a given degree of accuracy.

The " $T$ " law gives for the fundamental thermodynamic properties

$$
\begin{gathered}
\left(C_{P}\right)_{T}=c T^{3} \\
H_{T}-H_{0}=\int_{0}^{T}\left(C_{P}\right)_{T} d T=\frac{1}{4} c T^{4}=\frac{1}{4}\left(C_{P}\right)_{T} T \\
\cdot \dot{S_{T}}=\int_{0}^{T} \frac{\left(C_{P}\right)_{T}}{T} d T=\frac{1}{3} c T^{3}=\frac{1}{3}\left(C_{P}\right)_{T}
\end{gathered}
$$


where the constant $c$ is dependent on the substance involved. Equation (41) as written assumes the validity of the Third Law of Thermodynamics in the case in question.

According to the Debye theory the constant $c$ is given by

$$
c=\frac{12}{5} \pi^{4} R / \theta_{D}^{3}
$$

per gram-atom, where $\theta_{l}$, is the Debye characteristic temperature. However, $c$ is rarely evaluated from eq (42) independently of the heat-capacity data, because the " $T^{3}$ " law expressed by eq (39) and (42) is only approximate: for any given substance the value of $\theta_{l}$ depends on the property from which it is derived. It also varies with temperature when derived from a given property such as heat capacity, and the "T"3" law may "fail" well below the highest temperature at which it is desired to apply eq (39).

Let us use the $T$ in eq (39)-(41) to represent the temperature on any scale assumed to be the thermodynamic one. In what follows it will be assumed that extrapolation to $0 \mathrm{~K}$ using the " $T^{3}$ " law was from a temperature $a$ which is $T_{48}^{a}$ on the IPTS-48 and $T_{68}^{a}$ on the IPTS-68, that measurements of enthalpy giving heat capacity are available at the temperatures above temperature $a$, and that a temperature $b$ (where $T_{48}=T_{48}^{b}$ and $T_{68}=T_{68}^{b}$ ) is such that $T_{68}^{b}=T_{48}^{a}$ [analogous to eq (3)]. As before, it is desired to find the correction converting a thermodynamic property on the basis of IPTS-48 and at $T_{48}=T_{48}^{\prime}$ to the basis of IPTS-68 and at $T_{68}=T_{68}^{\prime \prime}=T_{48}^{\prime}$, where $T_{48}^{\prime} \geqslant T_{48}^{a}$ and $T_{68}^{\prime \prime} \geqslant T_{6 x}^{b}$. The temperature ranges of interest are represented by these various symbols in the schematic diagram of figure 3. It will be assumed that $c$ is assigned values such that the heat capacity given by eq (39) agrees with the experimental value of $\left(C_{P^{4}}^{48}\right)^{a}$ if IPTS-48 is the basis, and with that of $\left(C_{P}^{68}\right)^{b}$ if IPTS-68 is the basis.

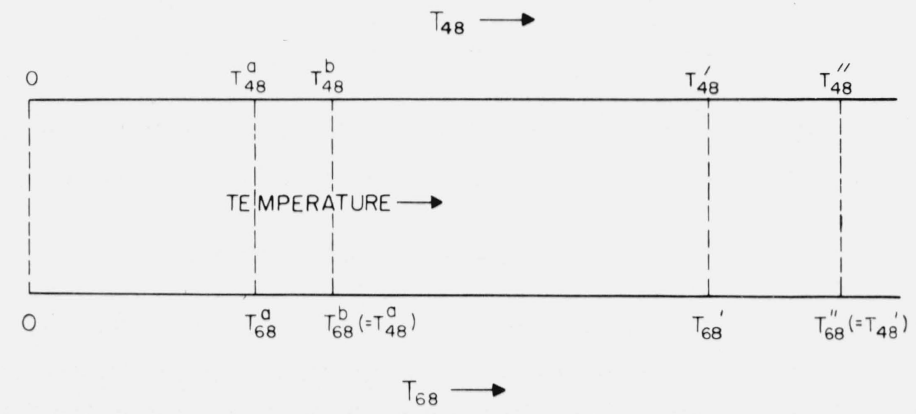

FIrURE 3. Temperature ranges of interest when extrapolation to $0 \mathrm{~K}$ was made using a theoretical relation (schematic).

As before, the desired correction to the enthalpy (this time specified to be relative to that at $0 \mathrm{~K}$ ) is $H^{\prime \prime}-H^{\prime}$, which can be found as the sum of the corrections to the enthalpy increment above $T_{68}^{b}\left(\right.$ or $\left.T_{48}^{a}\right)$ and the correction to the enthalpy increment below this temperature:

$$
\delta H=H^{\prime \prime}-H^{\prime} \equiv\left[\left(H^{\prime \prime}-H^{\prime}\right)-\left(H^{b}-H^{a}\right)\right]+\left[H^{b}-H^{a}\right] .
$$

The two parenthesized terms of eq (43) are separately calculable from eq (13), using the heatcapacity data on the IPTS -48 and $\mu\left(T_{68}\right)$. The second bracketed term in eq (43) is, from eq $(40)$,

$$
\begin{aligned}
{\left[H^{b}-H^{a}\right] } & =\frac{1}{4}\left(C_{P}^{68}\right)^{b} T_{68}^{b}-\frac{1}{4}\left(C_{P}^{48}\right)^{a} T_{48}^{a} \\
& =\frac{1}{4}\left[\left(C_{P}^{68}\right)^{b}-\left(C_{P}^{48}\right)^{a}\right] T_{48}^{a},
\end{aligned}
$$

since $T_{68}^{b}=T_{48}^{a} \cdot\left[\left(C_{P}^{68}\right)^{b}-\left(C_{P}^{48}\right)^{a}\right]$ is derivable from eq (16) after replacing temperatures 1 and 2 by $a$ and $b$ respectively. 
There is no modification to the heat capacity correction $\left[\left(C_{P}^{68}\right)^{\prime \prime}-\left(C_{P}^{48}\right)^{\prime}\right]$ given by eq (16) $\left(T_{68}^{\prime \prime} \geqslant T_{68}^{b}\right.$ and $\left.T_{48}^{\prime} \geqslant T_{48}^{a}\right)$, since the heat capacity in the experimental range is assumed to depend on temperature only in the manner observed experimentally.

As before, the desired correction to the entropy (taken as zero at $0 \mathrm{~K}$ because the Third Law of Thermodynamics is assumed) is $\left(S^{68}\right)^{\prime \prime}-\left(S^{48}\right)^{\prime}$, and

$$
\begin{aligned}
& \delta S=\left(S^{68}\right)^{\prime \prime}-\left(S^{48}\right)^{\prime} \equiv\left[\left(S^{68}\right)^{\prime \prime}-\left(S^{68}\right)^{b}\right] \\
& -\left[\left(S^{48}\right)^{\prime}-\left(S^{48}\right)^{a}\right]+\left[\left(S^{68}\right)^{b}-\left(S^{48}\right)^{a}\right] .
\end{aligned}
$$

From eq (9) (with subscripts changed correspondingly),

$$
T_{48}^{b}=T_{48}^{a}-\mu^{b}
$$

If eq (20) is modified by replacing the lower limit of integration by $T_{4 \times}^{b}$, given by eq (46), it becomes

$$
\left(S^{68}\right)^{\prime \prime}-\left(S^{68}\right)^{b}=\int_{T_{4 \times}^{u}-\mu^{b}}^{T_{4 \times}^{\prime}}\left(\frac{C_{P}^{48}}{T_{48}+\mu}\right) d T_{48}+\left(S^{68}\right)^{\prime \prime}-\left(S^{68}\right)
$$

where $\left(S^{68}\right)^{\prime \prime}-\left(S^{68}\right)^{\prime}$ is given, as before, by eq (34). Similarly, modifying the lower integration limit to $T_{48}^{a}$, eq (21) becomes

From eq (41),

$$
-\left[\left(S^{48}\right)^{\prime}-\left(S^{48}\right)^{a}\right]=-\int_{T_{4 \times}^{u}}^{T_{4 \times}^{\prime}} \frac{C_{P}^{48}}{T_{48}} d T_{48} .
$$

$$
\left[\left(S^{68}\right)^{b}-\left(S^{48}\right)^{a}\right]=\frac{1}{3}\left[\left(C_{P}^{68}\right)^{b}-\left(C_{P}^{48}\right)^{a}\right],
$$

where $\left.\left[C_{P^{\prime}}^{68}\right)^{b}-\left(C_{P}^{48}\right)^{a}\right]$ can be found from eq (16) for the two temperatures involved. The substitution from eq (47)-(49) into the last member of eq (45) then gives the desired entropy correction.

\subsection{Approximate Conversion Formulas}

Instead of using eq (13), (16), and (35) (or those in sec. 2.5) the enthalpy, heat capacity, and entropy may be corrected by the much simpler but approximate formulas below with sufficient accuracy for most purposes - even for the most accurate data except in the case of sharp transitions, and except possibly at very low temperatures. It should be realized, however, that the approximate formulas of this section may not be sufficiently accurate when applied to two temperature scales differing more widely from each other than the IPTS-48 and IPTS-68; in such cases, additional terms of the exact equations of the preceding sections are needed. Incidentally, it is well to point out that when the enthalpy data are sufficiently precise to justify the higherorder correction terms containing temperature derivatives of the heat capacity, it will often be simpler instead to repeat the treatment of the original unsmoothed data after replacing the boundary temperatures of each enthalpy increment in terms of the new temperature scale.

We define

$$
\delta X \equiv\left(X^{68}\right)_{T_{6 \times}=T_{0}}-\left(X^{48}\right)_{T_{4 \times}=T_{0}} .
$$

( $X \equiv C_{P}$, or $H$ or $S$ relative to that at a temperature where $\mu=0$. The superscript " 68 " or " 48 " indicates the temperature scale with respect to which the given value of $X$ was determined.)

$$
\mu \equiv T_{68}-T_{48}=t_{68}-t_{48}
$$

All properties are those at $T_{0}$, the nominal value of $T_{68}$ where the corrected property is desired, 
unless otherwise stated. The property values in the rhs of each equation below are as determined on the IPTS-48, and either $d T_{48}$ or $d T_{68}$ can be used for $d T$ [dropping the last term of eq (10)].

From eq (13), the only term of importance, usually, is the first, so

$$
\delta H=-\mu C_{P} .
$$

From eq (52),

$$
\delta C_{P}=\delta\left(\frac{d H}{d T}\right)=\frac{d}{d T} \delta H=-C_{P}, \frac{d \mu}{d T}-\mu \frac{d C_{P}}{d T} .
$$

Considering the correction to the absolute entropy, we can first write [using eq (53)]:

$$
\frac{d}{d T}(\delta S)=\delta\left(\frac{d S}{d T}\right)=\delta\left(\frac{C_{P}}{T}\right)=\frac{\delta C_{P}}{T}=-\frac{\mu}{T} \frac{d C_{P}}{d T}-\frac{C_{P}}{T} \frac{d \mu}{d T}
$$

But

$$
\frac{d}{d T}\left(-\int_{0}^{T} \frac{\mu C_{P} d T}{T^{2}}-\frac{\mu C_{P}}{T}\right) \equiv-\frac{\mu}{T} \frac{d C_{P}}{d T}-\frac{C_{P}}{T} \frac{d \mu}{d T}
$$

Since the last members of eq (54) and (55) are identical, we can write

$$
\delta S=-\int_{0}^{T} \frac{\mu C_{P} d T}{T^{2}}-\frac{\mu C_{P}}{T}
$$

of which the second member approximates the only important terms in eq (35).

The last member of eq (53) represents the only two terms of eq (16) that are usually of importance; and of these two terms, the first is probably nearly always the much more important (except, of course, when $d \mu / d T$ is accidentally very close to zero). According to this term, the relative (fractional) correction to the heat capacity is approximately equal in magnitude to $d \mu / d T$. According to table 4 , this does not exceed 0.3 percent below $3000 \mathrm{~K}$. The relative correction to the absolute entropy [eq (56)] tends to be considerably smaller, owing to partial cancellation within the integral and to the magnified weighting by the factor $1 / T^{2}$ of low temperatures where the differences between the IPTS-48 and the IPTS-68 are relatively small.

The corresponding equations that are accurate to the same approximation when the " $T^{3}$ " law is used below absolute temperature $\tau$, with the " $T$ " 3 " and experimental heat capacities required to agree at this temperature, are easily found from section 2.5. Thus, where $\delta H$ and $\delta S$ in the rhs are given by eq $(52)$ and (56) respectively:

$$
\begin{gathered}
\delta H=\frac{1}{4} \tau\left(\delta C_{P}\right)_{\tau}+(\delta H)-(\delta H)_{\tau} ; \\
\delta C_{P} \text { is given by eq }(53) ; \\
\delta S=\frac{1}{3}\left(\delta C_{P}\right)_{\tau}+(\delta S)-(\delta S)_{\tau} .
\end{gathered}
$$

\section{The Numerical Differences Between the International Practical Temperature Scales of 1948 and 1968}

The temperature ranges separately defined by the two scales are treated individually in sections 3.1-3.4 (headed by the respective temperature ranges expressed on the IPTS-68), and equations are derived giving numerically the differences $\mu\left(\equiv T_{68}-T_{48}=t_{68}-t_{48}\right)$ between the IPTS-68 and the IPTS-48 in these respective ranges, with sufficient accuracy for most practical purposes. 


\subsection{188 to $273.15 \mathrm{~K}\left(-182.962\right.$ to $\left.0^{\circ} \mathrm{C}\right)$}

The realization of both scales is specified to be by the resistance of a suitable platinum thermometer over the entire temperature range from approximately 90 to $904 \mathrm{~K}\left(-183\right.$ to $\left.631{ }^{\circ} \mathrm{C}\right)$. Since both scales are so defined as to preserve the highest practical continuity over this whole temperature range, their specifications below the ice point depend on those above the ice point. Therefore it will be necessary to consider together the definitions of the two scales in these two regions of temperature.

Using $W$ to represent the ratio of the measured resistance of a particular thermometer at some temperature to its resistance at $0{ }^{\circ} \mathrm{C},{ }^{4}$ the specified functions may be written:

IPTS-48 $\left(t_{48},-182.97\right.$ to $\left.0{ }^{\circ} \mathrm{C}\right)$ (Callendar-van Dusen equation) [4]:

$$
W=1+\alpha\left(1+0.01 \delta_{48}\right) t_{48}-10^{-4} \alpha \delta_{48} t_{48}^{2}-10^{-8} \alpha \beta t_{48}^{3}\left(100-t_{48}\right) .
$$

$\operatorname{IPTS}-68\left(t_{68},-182.962\right.$ to $\left.0^{\circ} \mathrm{C}\right)[1]$ :

$$
W=W_{1}^{*}+a_{4} t_{68}+c_{4} t_{68}^{3}\left(t_{68}-100\right) .
$$

IPTS-48 $\left(t_{48}, 0\right.$ to $\left.630.5^{\circ} \mathrm{C}\right)$ (Callendar equation) [4]:

$$
W=1+\alpha\left(1+0.01 \delta_{48}\right) t_{48}-10^{-4} \alpha \delta_{48} t_{48}^{2}
$$

IPTS-68 $\left(t_{6 \times}, 0\right.$ to $\left.630.74{ }^{\circ} \mathrm{C}\right)[1]$ :

which when rearranged is

$$
t^{\prime}=\frac{1}{\alpha}(W-1)+\delta_{68}\left(\frac{t^{\prime}}{100}\right)\left(\frac{t^{\prime}}{100}-1\right) .
$$

$$
W\left(t^{\prime}\right)=1+\alpha\left(1+0.01 \delta_{68}\right) t^{\prime}-10^{-4} \alpha \delta_{68}\left(t^{\prime}\right)^{2} .
$$

where $t^{\prime}$ is defined (as a monotonic function) by the equation

\begin{tabular}{|c|c|c|}
\hline \multirow{2}{*}{ Fixed point } & \multicolumn{2}{|c|}{ Temperature value } \\
\hline & IPTS-48 $[4,5]$ & IPTS-68 [1] \\
\hline 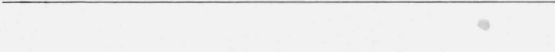 & ${ }^{\circ} \mathrm{C}$ & ${ }^{\circ} \mathrm{C}$ \\
\hline Normal boiling point of oxygen.............. & -182.970 & -182.962 \\
\hline Triple point of water............................. & 0.01 & 0.01 \\
\hline Normal boiling point of water................... & 100 & 100 \\
\hline Normal boiling point of sulfur................. & a 444.600 & \\
\hline Normal freezing point of zinc................... & " 419.505 & 419.58 \\
\hline
\end{tabular}

$$
t_{6 \times}=t^{\prime}+0.045\left(\frac{t^{\prime}}{100}\right)\left(\frac{t^{\prime}}{100}-1\right)\left(\frac{t^{\prime}}{419.58}-1\right)\left(\frac{t^{\prime}}{630.74}-1\right)
$$

The functions $W\left(t_{48}\right)$ and $W\left(t_{68}\right)$ differ somewhat from one thermometer to another, and the con-

TABLE 1. Fixed points used for calibration of platinum resistance thermometers using eq (60)-(64)

"For IPTS-48. the "sulfur" point was an official defining fixed point. but the substitution of the "zinc" peint and the temperature listed for it were recommended.

${ }^{4}$ Actually. a temoerature whose value is exactly $0{ }^{\circ} \mathrm{C}$ on one of the two scales has a value triviallv different (by $5 \times 10^{-6}{ }^{\circ} \mathrm{C}$ ) on the other scale.

${ }^{5}$ In ref. [1], $W_{1}^{*}$ is written as $W_{\text {(СТ. .6* }}\left(T_{\text {6к }}\right)$. 
- stants appearing in eq (60)-(63a) are determined for a particular thermometer by measuring $W$ at the fixed points listed in table $1 . W_{*}^{*}$. which appears in eq $(61)$, is a single-valued reference function given by the equation

$$
t_{68}=\sum_{i=1}^{20} A_{i}\left[\ln W_{i}^{*}\left(T_{6 \times}\right)\right]^{i},
$$

with the 20 constants $A_{1} \ldots A_{20}$ defined numerically and having 16 significant figures each [1].

Since the correction to the heat capacity is approximately proportional to $d \mu / d T$ [eq (53)], we shall be concerned as to whether the latter and its temperature derivative are continuous at $0{ }^{\circ} \mathrm{C}$, above and below which both $W\left(T_{48}\right)$ and $W\left(T_{68}\right)$ are each defined differently by the above equations. Among $W, T_{48}$, and $T_{68}$ there is only one independent variable, so we can write

$$
\frac{d \mu}{d T_{68}}=\frac{d\left(T_{68}-T_{48}\right)}{d T_{68}}=1-\frac{d T_{48}}{d T_{68}}=1-\frac{d W}{d T_{68}} / \frac{d W}{d T_{48}} .
$$

It is clear [from eq (60) and (62)] that $d W / d T_{48}$ and $d^{2} W / d T_{+\varsigma}^{2}$ are continuous at $0{ }^{\circ} \mathrm{C}$, but the situation for the corresponding derivatives involving $T_{68}$ is not obvious. From eq $(66), d \mu / d T_{68}$ and $d^{2} \mu / d T_{68}^{2}$ are continuous at $0^{\circ} \mathrm{C}$ if and only if $d W / d T_{68}$ and $d^{2} W / d T_{68}^{2}$ also are.

It turns out that the two derivatives of $W$ involving $T_{68}$ as defined by eq (61), (63a), and (64) are not identically continuous at $0{ }^{\circ} \mathrm{C}$. The statement has been made, however, that they are continuous at $0{ }^{\circ} \mathrm{C}$ for what may be regarded as a typical thermometer having the following constants [1] (using asterisks for this special case):

$$
a_{4}^{*}=c_{4}^{*}=0 ; \alpha^{*}=3.9259668\left(10^{-3}\right) ; \delta_{6 \mathrm{~s}}^{*}=1.496334 .
$$

This continuity may be readily demonstrated, as shown below. Incidentally, the corresponding function $W^{*}\left(T_{68}\right)\left[\equiv W_{t}^{*}\right.$ at and below $0{ }^{\circ} \mathrm{C}$; $\equiv W^{*}\left(t^{\prime}\right)$ at and above $\left.0{ }^{\circ} \mathrm{C}\right]$ may alternatively be regarded as the standard reference function defining $t_{\text {tix }}$ from -182.962 to $630.74{ }^{\circ} \mathrm{C}$.

Since $W_{i}^{*}=1$ at $T_{68}=273.15$, it was found, using eq (65) and the numerical values of the constants $A_{i}$, that at $0{ }^{\circ} \mathrm{C}$

$$
\frac{d W_{i}^{*}}{d T_{68}}=1 / \frac{d T_{68}}{d W_{i}^{*}}=\frac{1}{A_{1}}=3.98650632\left(10^{-3}\right)
$$

and

$$
\frac{d^{2} W_{1}^{*}}{d T^{*}{ }_{68}}=-\frac{d^{2} T_{68}}{d W_{1}^{* 2}} /\left(\frac{d T_{68}}{d W_{1}^{*}}\right)^{3}=\frac{A_{1}-2 A_{2}}{A_{1}^{3}}=-1.2261330\left(10^{-6}\right) .
$$

Similarly, it was found, using eq $(63 \mathrm{a}),(64)$, and $(67)$, that at $0{ }^{\circ} \mathrm{C}$

$$
\frac{d W^{*}\left(t^{\prime}\right)}{d T_{68}}=\frac{d W^{*}\left(t^{\prime}\right)}{d t^{\prime}} / \frac{d t_{68}}{d t^{\prime}}=3.98650630\left(10^{-3}\right)
$$

and

$\frac{d^{2} W^{*}\left(t^{\prime}\right)}{d T_{68}^{2}}=\frac{d^{2} W^{*}\left(t^{\prime}\right)}{d t^{\prime 2}} /\left(\frac{d t_{68}}{d t^{\prime}}\right)^{2}-\frac{d W^{*}\left(t^{\prime}\right)}{d t^{\prime}}\left(\frac{d^{2} t_{68}}{d t^{\prime 2}}\right) /\left(\frac{d t_{68}}{d t^{\prime}}\right)^{3}=-1.2261327\left(10^{-6}\right)$.

The values of eq (68) and (70) differ by a factor of $1+5\left(10^{-9}\right)$, and those of eq (69) and (71) by a factor of $1+2\left(10^{-7}\right)$. Though the pertinent magnitudes are such that at $0^{\circ} \mathrm{C}$ the fractional discontinuity in $d \mu / d T_{68}$ is many times as great as that in $d W / d T_{68}$, these discrepancies are too small to be of any practical significance and are very likely to be so for all resistance thermometers meeting the standards imposed by the IPTS -68 . 
For a given resistance thermometer, $\alpha$ has the same value in eq (60), (62), (63), and (63a), because it depends only on the resistances at 0 and $100{ }^{\circ} \mathrm{C}$, which designate the same ${ }^{4}$ (see footnote 4) respective temperatures on the IPTS-48 and the IPTS-68. However, $\delta_{48} \neq \delta_{68}$, because of the difference in the two values of temperature for the zinc (or sulfur) point (table 1). At the zinc point $t_{48}=419.505$ and $t^{\prime}=t_{68}=419.58$. Substituting these values and that of $\delta_{68}^{*}$ from eq (67) into eq (62) and (63a) and equating the two expressions for $W$ give

$$
\delta_{48}^{*}=1.49135723
$$

Since $W$ also is a continuous function of temperature, $W$ and its derivatives may replace $H$ and its derivatives in eq (13) to give an equation from which $\mu\left(t_{68}\right)$ may be derived,

$$
W^{\prime \prime}-W^{\prime}=-\mu^{\prime \prime}\left(\frac{d W}{d t_{48}}\right)^{\prime}+\frac{1}{2}\left(\mu^{\prime \prime}\right)^{2}\left(\frac{d^{2} W}{d t^{2} \times}\right)^{\prime}-\ldots
$$

In rhs of eq (73) all terms except the first may with sufficient accuracy be neglected (the ratio of the second term to the first does not exceed 0.00004 between -182.962 and $630.74{ }^{\circ} \mathrm{C}$ ), giving

$$
\mu^{\prime \prime}=\left(W^{\prime}-W^{\prime \prime}\right) /\left(\frac{d W}{d t_{48}}\right)^{\prime}
$$

Using for $W$ the standard reference function defined by eq (60)-(65) with the parameter values of eq (67) and (72), we shall derive from eq (74) numerical functions for $\mu\left(t_{68}\right)$, in this section for the range $t_{68}=-182.962$ to $0{ }^{\circ} \mathrm{C}$ and in the next section for the range $t_{68}=0$ to $630.74{ }^{\circ} \mathrm{C}$.

Since eq (74) expresses $\mu$ at temperature 2 , for temperatures at or below $0{ }^{\circ} \mathrm{C} W^{\prime}$ and $\left(d W / d t_{48}\right)^{\prime}$ are found from eq $(60),(67)$, and (72), then replacing $t_{48}^{\prime}$ by $t_{68}^{\prime \prime}$ by eq (1)-(3), and $W^{\prime \prime}$ from eq (61) and (67). [The value of $\beta$ is found from eq (60) applied at the oxygen boiling point, where $W$ has the value of $W_{t}^{*}$ at $T_{68}=90.188 \mathrm{~K}$ and where $t_{48}=-182.970$ (table 1).] The final equation is

$$
\begin{gathered}
\mu=\frac{250.97\left[1+3.9845170\left(10^{-3}\right) t_{68}-5.855019\left(10^{-7}\right) t_{68}^{2}+4.35717\left(10^{-12}\right)\left(100-t_{68}\right) t_{68}^{3}-W_{t}^{*}\right]}{1-2.9389\left(10^{-4}\right) t_{68}+4.3741\left(10^{-9}\right)\left(75-t_{68}\right) t_{68}^{2}} \\
{\left[t_{68}=-182.962 \text { to } 0^{\circ} \mathrm{C}\right] .}
\end{gathered}
$$

\subsection{15 to $903.89 \mathrm{~K}\left(0\right.$ to $\left.630.74^{\circ} \mathrm{C}\right)$}

For temperatures at and above $0{ }^{\circ} \mathrm{C}$, eq (74) is applied similarly as was done in section 3.1 for the lower temperature range. $W^{\prime}$ and $\left(d W / d t_{48}\right)^{\prime}$ are found from eq $(62)$, replacing $t_{48}^{\prime}$ by $t_{68}^{\prime \prime}$, and $W^{\prime \prime}$ from eq $(63 \mathrm{a})$. For replacing $t^{\prime}$ in eq (63a) in terms of $t_{6 \times}$, eq (64) may be written

where

$$
t_{68}=t^{\prime}+\varphi\left(t^{\prime}\right)
$$

$$
\varphi\left(t^{\prime}\right) \equiv 0.045\left(\frac{t^{\prime}}{100}\right)\left(\frac{t^{\prime}}{100}-1\right)\left(\frac{t^{\prime}}{419.58}-1\right)\left(\frac{t^{\prime}}{630.74}-1\right) .
$$

Expanding $\varphi\left(t^{\prime}\right)$ to two terms about $\varphi\left(t_{68}\right)$ and then replacing $\varphi\left(t^{\prime}\right)$ from eq (76) gives

$$
t^{\prime}=t_{68}-\frac{\varphi\left(t_{68}\right)}{1+\frac{d \varphi\left(t_{68}\right)}{d t_{68}}} \text {. }
$$

(The magnitude of $d \varphi\left(t_{68}\right) / d t_{68}$ never exceeds about 0.001.) Neglecting a term [in $\varphi\left(t_{68}\right)$ ] whose magnitude never exceeds approximately $0.00002{ }^{\circ} \mathrm{C}$, the result is 


$$
\mu=\frac{0.01\left(\delta_{68}-\delta_{48}\right) t_{68}\left(0.01 t_{68}-1\right)}{1+0.01 \delta_{48}-0.0002 \delta_{48} t_{68}}+\varphi\left(t_{68}\right)
$$

Substituting the values of $\delta_{68}$ and $\delta_{48}$ for the reference function [eq (67) and (72)] gives the final numerical equation,

$$
\mu=\frac{4.904\left(10^{-7}\right) t_{68}\left(t_{68}-100\right)}{1-2.939\left(10^{-4}\right) t_{68}}+\varphi\left(t_{68}\right), \quad\left[t_{68}=0 \text { to } 630.74{ }^{\circ} \mathrm{C}\right]
$$

where $\varphi\left(t_{68}\right)$ is given by eq (77) on replacing $t^{\prime}$ by $t_{68 .}$. Differentiation of eq (80) gives

$$
\begin{gathered}
\frac{d \mu}{d t_{68}}=\frac{-4.904\left(10^{-5}\right)\left[1-0.02 t_{68}+2.939\left(10^{-6}\right) t_{68}^{2}\right]}{\left[1-2.939\left(10^{-4}\right) t_{68}\right]^{2}}+\frac{d \varphi\left(t_{68}\right)}{d t_{68}} . \\
{\left[t_{68}=0 \text { to } 630.74^{\circ} \mathrm{C}\right]}
\end{gathered}
$$

\subsection{89 to $1,337.58 \mathrm{~K}\left(630.74\right.$ to $\left.1,064.43{ }^{\circ} \mathrm{C}\right)$}

Temberature in this range is measured by a platinum-versus-rhodium-platinum thermocouple of suitable specifications, the temperature being defined on the IPTS-48 [4] by

$$
E=a^{\prime}+b^{\prime} t_{48}+c^{\prime} t_{48}^{2},
$$

and on the IPTS-68 [1] by

$$
E=a+b t_{68}+c t_{68}^{2} .
$$

In these equations $E$ is the electromotive force of the thermocouple, and the six constants $a^{\prime} \ldots c$ are evaluated by calibration at the three fixed points listed in table 2 . The IPTS- 68 specifies that $E$ shall have the respective values listed in the last column of table 2, within specified tolerances. We may regard these nominal values as defining for each of the two scales a "standard" emftemperature function to which the emf of a given thermocouple may be corrected through its calibration.

The right-hand sides of eq (82) and (83) may be equated, then $t_{48}$ replaced by $\left(t_{68}-\mu\right)$ [eq (1), (2), and (6)]. The result on solving for $\mu$ may be written

$$
\mu=\frac{\left(a^{\prime}-a\right)+\left(b^{\prime}-b\right) t_{68}+\left(c^{\prime}-c\right) t_{68}^{2}}{b^{\prime}+2 c^{\prime} t_{68}-c^{\prime} \mu} .
$$

The term $-c^{\prime} \mu$ in the denominator is virtually negingible, but $\mu$ in this term may be replaced by $-1.616+0.002864 t_{68}$, which fits the three values of table 2 within $0.01{ }^{\circ} \mathrm{C}$. After using the values

TABLE 2. Fixed points used for calibration of $\mathrm{Pt} / \mathrm{Pt}-10 \%$ Rh thermocouples using eq (82) and (83)

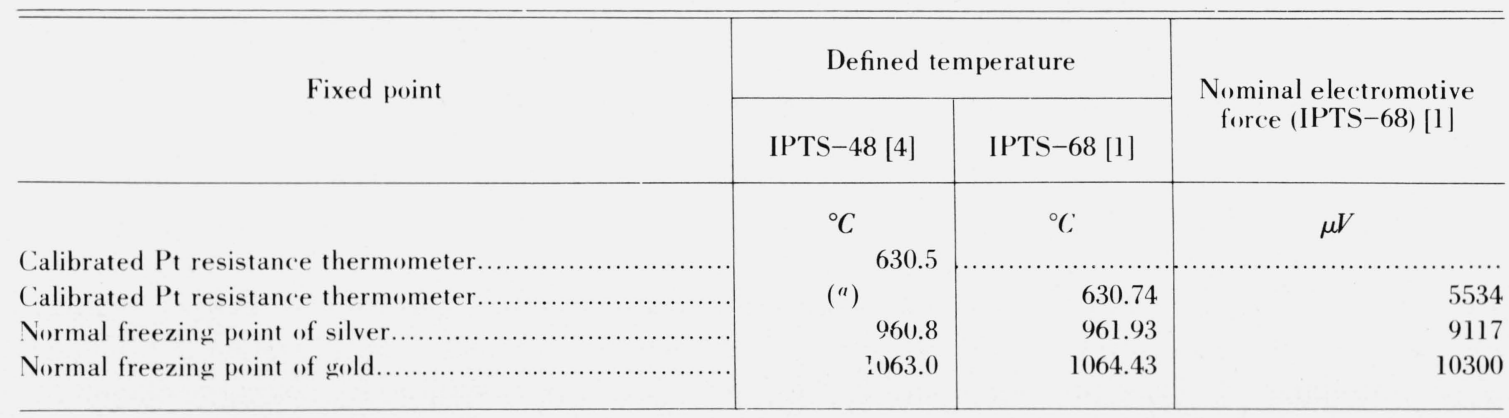

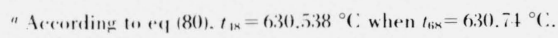


of temperature and electromotive force listed in table 2 to solve eq (82) and (83) for the six constants $a^{\prime} \ldots c$, their substitution into eq (84) gives the numerical equation

$$
\begin{aligned}
& \mu=\frac{-1.3145+1.5016\left(10^{-3}\right) t_{68}+1.5625\left(10^{-6}\right) t_{68}^{2} .}{1+4.101\left(10^{-4}\right) t_{68}} \\
& \quad\left[t_{68}=630.74 \text { to } 1064.43^{\circ} \mathrm{C}\right]
\end{aligned}
$$

Differentiation of eq (85) gives

$$
\begin{gathered}
\frac{d \mu}{d t_{68}}=\frac{2.0407\left(10^{-3}\right)\left[1+1.53135\left(10^{-3}\right) t_{68}+3.1400\left(10^{-7}\right) t_{68}^{2}\right]}{\left[1+4.101\left(10^{-4}\right) t_{68}\right]^{2}} \\
{\left[t_{68}=630.74 \text { to } 1064.43^{\circ} \mathrm{C}\right]}
\end{gathered}
$$

\section{4. $1,337.58 \mathrm{~K}\left(1,064.43{ }^{\circ} \mathrm{C}\right)$ to $10,000 \mathrm{~K}$}

Above the freezing point of gold both the IPTS-48 and the IPTS-68 define the temperature by the Planck Radiation Law, according to which, at wavelength $\lambda$ and in the range $d \lambda$, the ratio of the spectral intensity at the absolute thermodynamic temperature $T$ to that at the absolute themodynamic temperature of freezing gold, $T(\mathrm{Au})$, is

$$
\frac{\exp \left[\frac{c_{2}}{\lambda T(\mathrm{Au})}\right]-1}{\exp \left[\frac{c_{2}}{\lambda T}\right]-1},
$$

where $c_{2}$ is the so-called second radiation constant. The definition of $T_{48}$ is obtained by making the replacements $T=T_{48}, c_{2}=1.438 \mathrm{~cm}$ kelvin, and $T(\mathrm{Au})=1336.15 \mathrm{~K}$ in (87) [4], and the definition of $T_{68}$ by the substitutions $T=T_{68}, c_{2}=1.4388 \mathrm{~cm}$ kelvin, and $T(\mathrm{Au})=1337.58 \mathrm{~K}$ [1]. These functions for the two scales may, of course, be equated; when the equation is solved for $T_{48}$ and this then subtracted from $T_{68}$, it gives $T_{68}-T_{48}$, or $\mu\left(T_{68}\right)$, exactly. A simpler numerical equation results by expanding some of the exponentials and dropping negligible terms; when the representative wavelength of optical pyrometers $\lambda=6.5\left(10^{-5}\right) \mathrm{cm}$ is substituted, the result is

$$
\begin{aligned}
\mu=5.56\left(10^{-4}\right) T_{68}+3.84\left(10^{-7}\right)\left(1-e^{-22135 / T_{68}}\right) T_{68}^{2} \\
{\left[T_{68}=1,337.58 \text { to } 10,000\right] }
\end{aligned}
$$

Up to $10,000 \mathrm{~K}$ eq (88) is insensitive to wavelength, and the error in $\mu$ does not exceed about 0.1 kelvin.

Differentiation of eq (88) gives

$$
\begin{array}{r}
\frac{d \mu}{d T_{68}}=5.56\left(10^{-4}\right)+7.68\left(10^{-7}\right) T_{68}-\left[8.5\left(10^{-3}\right)+7.68\left(10^{-7}\right) T_{68}\right] e^{-22135 / T_{68}} \\
{\left[T_{68}=1,337.58 \text { to } 10,000\right]}
\end{array}
$$

\subsection{Discontinuities in the Temperature Derivatives of $t_{68}-t_{48}$ at 630.74 and $1,064.43{ }^{\circ} \mathrm{C}$}

Though both the IPTS-48 and the IPTS-68 treat the "resistance thermometer" temperature range differently below and above $0{ }^{\circ} \mathrm{C}$, the two scales are highly continuous at this temperature and no discontinuity in $t_{68}-t_{48}(=\mu)$ and its first two temperature derivatives exists, as demonstrated in section 3.1. This is not true where the "thermometer" and "thermocouple" ranges join $\left(630.74{ }^{\circ} \mathrm{C}\right)$, nor where the "thermocouple" and "pyrometer" ranges join $\left(1,064.43{ }^{\circ} \mathrm{C}\right)$. Though $\mu$ is continuous at these two temperatures, $d \mu / d t_{68}$ is not, the discontinuity having a magnitude of about 0.001 in both cases. 
TABLE 3. Comparison of the difference in the value of temperature, and its temperature derivative, given by the IPTS of 1968 and the IPTS of 1948 (a) as the two scales are defined and (b) as smoothed in the regions of discontinuity.

\begin{tabular}{|c|c|c|c|c|}
\hline \multirow{2}{*}{$T_{6 \times 8}(K)$} & \multicolumn{2}{|c|}{$T_{6 ; \times}-T_{4 \times}$ (kelvins) } & \multicolumn{2}{|c|}{$d\left(T_{6 \times}-T_{48}\right) / d T_{68}$} \\
\hline & As defined & Smoothed & As defined & Smoothed \\
\hline 840 & $\mathrm{a}+0.113$ & & $\mathrm{f}+0.0009$ & \\
\hline 850 & .123 & $\mathrm{~d}+0.123$ & .0010 & $\mathrm{~d}+0.0011$ \\
\hline 860 & .134 & .135 & .0012 & .0013 \\
\hline 870 & .146 & .150 & .0013 & .0016 \\
\hline 880 & .160 & .166 & .0015 & .0018 \\
\hline 890 & .176 & .185 & .0017 & .0020 \\
\hline 900 & ${ }^{a} .194$ & .205 & .0019 & .0021 \\
\hline 903.89 & a, b .202 & .214 & $\left\{\begin{array}{l}f .0020 \\
f .0027\end{array}\right\}$ & .0022 \\
\hline 910 & ' .218 & .227 & .0027 & .0023 \\
\hline 920 & .245 & .251 & .0027 & .0024 \\
\hline 940 & .300 & .301 & .0027 & .0026 \\
\hline 960 & .354 & d .354 & .0027 & ${ }^{\mathrm{d}} .0027$ \\
\hline 980 & b. .409 & & ".0027 & \\
\hline 1260 & b) 1.202 & & ‥0029 & .......... \\
\hline 1280 & 1.261 & " 1.261 & .0029 & ".0029 \\
\hline 1300 & 1.319 & 1.316 & .0029 & .0027 \\
\hline 1320 & " 1.378 & 1.368 & .0029 & .0025 \\
\hline 1337.58 & b. e 1.430 & 1.410 & $\left\{\begin{array}{l}{ }^{k} .0030 \\
h^{h} .0016\end{array}\right\}$ & .0023 \\
\hline 1350 & " 1.45 & 1.44 & .0016 & .0022 \\
\hline 1375 & 1.49 & e 1.49 & .0016 & ".0019 \\
\hline 1400 & ' 1.53 & & h. .0016 & $\ldots \ldots \ldots$ \\
\hline
\end{tabular}

Though these discontinuities reflect small corresponding discontinuities at these two temperatures in the IPTS -48 or the IPTS-68 or both, it is not the purpose here to discuss this question. The principal additional discontinuity introduced when calorimetric thermodynamic properties are converted from the basis of one temperature scale to that of the other is when the heat capacitytemperature function has been smoothed prior to this conversion: the conversion will introduce an approximately 0.1 percent discontinuity. Such an effect is virtually within the precision of the best present-day calorimetry at temperatures as high as $631{ }^{\circ} \mathrm{C}$, but it may be desirable to avoid it in the systematic conversion of many existing tables.

An obvious simple expedient is to replace the functions $\mu\left(t_{68}\right)$, over a temperature range extending below and above each temperature of discontinuity, by a function which provides continuity with the pertinent original functions in both $\mu$ and $d \mu / d t_{68}$ at the ends of the modified range. That the extent of such a modified range is normally an arbitrary decision is evident from consideration of the idealized simple case (sometimes approximated in reality) in which the heat capacity shows a discontinuity at one temperature but otherwise no change with temperature: How should one join the two disconnected segments smoothly? However, when the effect is as small as in the present case, this question can hardly be important.

The procedure outlined above was followed to derive simple polynomial "smooth" equations for the temperature ranges 570 to $690{ }^{\circ} \mathrm{C}$ and 1000 to $1120^{\circ} \mathrm{C}$. These equations are as follows. 


$$
\begin{array}{rlr}
\mu= & 0.1162+9.153\left(10^{-4}\right)\left(t_{68}-570\right)+1.3328\left(10^{-5}\right)\left(t_{68}-570\right)^{2} \\
& -3.192\left(10^{-8}\right)\left(t_{68}-570\right)^{3} & {\left[t_{68}=570 \text { to } 690{ }^{\circ} \mathrm{C}\right]} \\
\mu= & 1.241+2.92\left(10^{-3}\right)\left(t_{68}-1000\right)-4.092\left(10^{-6}\right)\left(t_{68}-1000\right)^{2} \\
& -7.222\left(10^{-9}\right)\left(t_{68}-1000\right)^{3} & {\left[t_{68}=1000 \text { to } 1120{ }^{\circ} \mathrm{C}\right]}
\end{array}
$$

These two equations and their first derivatives are compared in table 3 with the equations which it is proposed they displace in the temperature ranges indicated above when converting values that were previously smoothed with respect to temperature on the IPTS-48. When no such smoothing was done, eq (90) and (91) should not be used.

\subsection{Tabulated Values of $\left(T_{68}-T_{48}\right)$ and $d\left(T_{68}-T_{48}\right) / d T_{68}$ at Round Temperatures of $T_{68}$ From 90 to $10,000 \mathrm{~K}$}

Values of $T_{68}-T_{48}(\equiv \mu)$ and $d\left(T_{68}-T_{48}\right) / d T_{68} \quad\left(=d \mu / d t_{68}\right)$, computed from the equations derived in sections 3.1-3.4 [ $\mu$ from eq (75), (80), (85), and (88); $d \mu / d t_{68}$ from eq (81). (86), and (89)], are tabulated at round temperatures $\left(T_{68}\right)$ in table 4 . Below $0{ }^{\circ} \mathrm{C}, d\left(T_{68}-T_{48}\right) / d T_{68}$ was taken by averaging successive pairs of differences of values of $T_{68}-T_{48}$ tabulated at intervals of 1 kelvin. Double entries are included for those temperatures for which the equations for the adjacent temperature ranges give different values (see discussion in sec. 3.5 ).

A similar table of $T_{68}-T_{48}$ covering the range -180 to $4000{ }^{\circ} \mathrm{C}$ has been published [1]. The two tables agree exactly, to the number of significant figures given in each.

\begin{tabular}{|c|c|c|c|c|c|c|c|c|}
\hline$T_{68}$ & $T_{68 x}-T_{48}$ & $\left|d\left(T_{68}-T_{4 \times}\right) / d T_{6 \times}\right|$ & $T_{68}$ & $T_{68}-T_{48}$ & $d\left(T_{68}-T_{48}\right) / d T_{68}$ & $T_{6 \mathrm{x}}$ & $T_{68}-T_{48}$ & $d\left(T_{68}-T_{48}\right) / d T_{68}$ \\
\hline 90 & +0.0076 & +0.0022 & 128 & -0.0135 & 0.00000 & 220 & +0.0299 & -0.00038 \\
\hline 90.188 & .0080 & .0021 & 130 & -.0134 & +.00015 & 225 & .0278 & -.00045 \\
\hline 91 & .0095 & .0017 & 132 & -.0129 & .00028 & 230 & .0254 & -.00051 \\
\hline 92 & .0110 & .0013 & 134 & -.0122 & .00041 & 235 & .0227 & -.00056 \\
\hline 93 & .0121 & .0008 & 136 & -.0113 & .00052 & 240 & .0198 & -.00059 \\
\hline 94 & .0127 & .0005 & 138 & -.0102 & .00062 & 245 & .0168 & -.00061 \\
\hline 95 & .0130 & .0002 & 140 & -.0088 & .00071 & 250 & .0137 & -.00062 \\
\hline 96 & .0130 & -.0001 & 142 & -.0073 & .00079 & 255 & .0105 & -.00062 \\
\hline 97 & .0128 & -.0004 & 144 & -.0057 & .00086 & 260 & .0074 & -.00061 \\
\hline 98 & .0123 & -.0006 & 146 & -.0039 & .00092 & 265 & .0044 & -.00058 \\
\hline 99 & .0116 & -.0008 & 148. & -.0020 & .00096 & 270 & .0016 & -.00054 \\
\hline 100 & .0108 & -.00089 & 150 & .0000 & .00100 & 273.15 & .0000 & -.00050 \\
\hline 102 & .0088 & -.00112 & 155 & +.0050 & .00103 & 275 & -.0009 & -.00047 \\
\hline 104 & .0064 & -.00124 & 160 & .0102 & .00102 & 280 & -.0031 & -.00041 \\
\hline 106 & .0038 & -.00130 & 165 & .0152 & .00096 & 285 & -.0050 & -.00035 \\
\hline 108 & .0012 & -.00130 & 170 & .0197 & .00087 & 290 & -.0066 & -.00029 \\
\hline 110 & -.0013 & -.00125 & 175 & .0238 & .00075 & 295 & -.0079 & -.00023 \\
\hline 112 & -.0038 & -.00116 & 180 & .0272 & .00061 & 298.15 & -.0085 & -.00020 \\
\hline 114 & -.0060 & -.00106 & 185 & .0299 & .00047 & 300 & -.0089 & -.00018 \\
\hline 116 & -.0080 & -.00092 & 190 & .0318 & .00032 & 305 & -.0096 & -.00013 \\
\hline 118 & -.0097 & -.00078 & 195 & .0331 & .00017 & 310 & -.0101 & -.00008 \\
\hline 120 & -.0111 & -.00063 & 200 & .0336 & .00005 & 315 & -.0104 & -.00003 \\
\hline 122 & -.0122 & -.00046 & 205 & .0335 & -.00009 & 320 & -.0105 & +.00001 \\
\hline 124 & -.0129 & -.00030 & 210 & .0328 & -.00020 & 325 & -.0103 & .00006 \\
\hline 126 & -.0134 & -.00015 & 215 & .0316 & -.00029 & 330 & -.0099 & .00009 \\
\hline
\end{tabular}

TABLE 4. Approximate difference in the value of temperature, and its temperature derivative, given by the International Practical Temperature Scales of 1968 and 1948

(The unit of temperature is the kelvin. For both scales, $T(K)=t\left({ }^{\circ} \mathrm{C}:\right)+273.15$.) 
TABle 4. Approximate difference in the value of temperature, and its temperature derivative, given by the International Practical Temperature Scales of 1968 and 1948 - Continued

(The unit of temperature is the kelvin. For both scales, $T(K)=t\left({ }^{\circ} \mathrm{C}\right)+273.15$.)

\begin{tabular}{|c|c|c|c|c|c|c|c|c|}
\hline$T_{68}$ & $T_{68}-T_{48}$ & $d\left(T_{68}-T_{48}\right) / d T_{68}$ & $T_{68}$ & $T_{68}-T_{48}$ & $d\left(T_{68}-T_{48}\right) / d T_{68}$ & $T_{68}$ & $T_{68}-T_{48}$ & $d\left(T_{68}-T_{48}\right) / d T_{68}$ \\
\hline 335 & -0.0093 & +0.00013 & 700 & +0.075 & 0.0000 & 1320 & +1.378 & +0.0029 \\
\hline 340 & -.0086 & .00016 & 710 & .074 & .0000 & & & $\int^{b} .0030$ \\
\hline 345 & -.0077 & .00020 & 720 & .074 & .0000 & 1337.58 & 1.430 & $\left\{{ }^{c} .0016\right.$ \\
\hline 350 & -.0066 & .00023 & 730 & .074 & .0000 & 1350 & 1.45 & .0016 \\
\hline 355 & -.0054 & .00026 & 740 & .075 & +.0001 & 1375 & 1.49 & .0016 \\
\hline 360 & -.0041 & .00028 & 750 & .076 & .0001 & 1400 & 1.53 & .0016 \\
\hline 365 & -.0026 & .00030 & 760 & .077 & .0001 & 1425 & 1.57 & .0017 \\
\hline 370 & -.0010 & .00033 & 770 & .079 & .0002 & 1450 & 1.61 & .0017 \\
\hline 373.15 & .0000 & .00034 & 780 & .081 & .0003 & 1475 & 1.66 & .0017 \\
\hline 375 & +.0006 & .00035 & 790 & .084 & .0003 & 1500 & 1.70 & .0017 \\
\hline 380 & .0024 & .00036 & 800 & .088 & .0004 & 1525 & 1.74 & .0017 \\
\hline 385 & .0043 & .00038 & 810 & .093 & .0005 & 1550 & 1.78 & .0017 \\
\hline 390 & .0062 & .00039 & 820 & .098 & .0006 & 1575 & 1.83 & .0018 \\
\hline 395 & .0082 & .00041 & 830 & .105 & .0007 & 1600 & 1.87 & .0018 \\
\hline 400 & .0103 & .00042 & 840 & .113 & .0009 & 1625 & 1.92 & .0018 \\
\hline 410 & .0146 & .00044 & 850 & .123 & .0010 & 1650 & 1.96 & .0018 \\
\hline 420 & .0190 & .00045 & 860 & .134 & .0012 & 1675 & 2.01 & .0018 \\
\hline 430 & .0235 & .00045 & 870 & .146 & .0013 & 1700 & 2.05 & .0019 \\
\hline 440 & .0280 & .00045 & 880 & .160 & .0015 & 1725 & 2.10 & .0019 \\
\hline 450 & .0325 & .00045 & 890 & .176 & .0017 & 1750 & 2.15 & .0019 \\
\hline 460 & .0369 & .00044 & 900 & .194 & .0019 & 1775 & 2.20 & .0019 \\
\hline 470 & .0413 & .00043 & & & a a .0020 & 1800 & 2.24 & .0019 \\
\hline 480 & .0454 & .00041 & 903.89 & .202 & $\left\{{ }^{b} .0027\right.$ & 1825 & 2.29 & .0020 \\
\hline 490 & .0494 & .00039 & 910 & .218 & .0027 & 1850 & 2.34 & .0020 \\
\hline 500 & .0532 & .00037 & 920 & .245 & .0027 & 1875 & 2.39 & .0020 \\
\hline 510 & .057 & .0003 & 940 & .300 & .0027 & 1900 & 2.44 & .0020 \\
\hline 520 & .060 & .0003 & 960 & .354 & .0027 & 1925 & 2.49 & .0020 \\
\hline 530 & .063 & .0003 & 980 & .409 & .0027 & 1950 & 2.54 & .0021 \\
\hline 540 & .066 & .0003 & 1000 & .464 & .0028 & 1975 & 2.60 & .0021 \\
\hline 550 & .068 & .0002 & 1020 & .519 & .0028 & 2000 & 2.65 & .0021 \\
\hline 560 & .070 & .0002 & 1040 & .575 & .0028 & 2050 & 2.75 & .0021 \\
\hline 570 & .072 & .0002 & 1060 & .631 & .0028 & 2100 & 2.86 & .0022 \\
\hline 580 & .074 & .0001 & 1080 & .687 & .0028 & 2150 & 2.97 & .0022 \\
\hline 590 & .075 & .0001 & 1100 & .743 & .0028 & 2200 & 3.08 & .0022 \\
\hline 600 & .076 & .0001 & 1120 & .800 & .0028 & 2250 & 3.19 & .0023 \\
\hline 610 & .076 & .0001 & 1140 & .857 & .0028 & 2300 & 3.31 & .0023 \\
\hline 620 & .077 & .0000 & 1160 & .914 & .0029 & 2350 & 3.43 & .0024 \\
\hline 630 & .077 & .0000 & 1180 & .971 & .0029 & 2400 & 3.55 & .0024 \\
\hline 640 & .077 & .0000 & 1200 & 1.029 & .0029 & 2450 & 3.67 & .0024 \\
\hline 650 & .077 & .0000 & 1220 & 1.086 & .0029 & 2500 & 3.79 & .0025 \\
\hline 660 & .076 & .0000 & 1235.08 & 1.130 & .0029 & 2600 & 4.0 & .003 \\
\hline 670 & .076 & .0000 & 1240 & 1.144 & .0029 & 2700 & 4.3 & .003 \\
\hline 680 & .076 & .0000 & 1260 & 1.202 & .0029 & 2800 & 4.6 & .003 \\
\hline 690 & .075 & .0000 & 1280 & 1.261 & .0029 & 2900 & 4.8 & .003 \\
\hline 692.73 & .075 & .0000 & 1300 & 1.319 & .0029 & 3000 & 5.1 & .003 \\
\hline
\end{tabular}


TABLE 4. Approximate difference in the value of temperature, and its temperature derivative, given by the International Practical Temperature Scales of 1968 and 1948-Continued

(The unit of temperature is the kelvin. For both scales, $T(K)=t\left({ }^{\circ} \mathrm{C}\right)+273.15$.)

\begin{tabular}{|c|c|c|c|c|c|c|c|c|}
\hline$T_{68}$ & $T_{68}-T_{48}$ & $d\left(T_{68}-T_{48}\right) / d T_{68}$ & $T_{68}$ & $T_{68}-T_{48}$ & $d\left(T_{68}-T_{48}\right) / d T_{68}$ & $T_{68}$ & $T_{68}-T_{48}$ & $d\left(T_{68}-T_{48}\right) / d T_{68}$ \\
\hline 3100 & +5.4 & +0.003 & 4100 & +8.7 & +0.004 & 5500 & +14 & +0.005 \\
\hline 3200 & 5.7 & .003 & 4200 & 9.1 & .004 & 6000 & 17. & .005 \\
\hline 3300 & 6.0 & .003 & 4300 & 9.4 & .004 & 6500 & 19. & .005 \\
\hline 3400 & 6.3 & .003 & 4400 & 9.8 & .004 & 7000 & 22. & .005 \\
\hline 3500 & 6.6 & .003 & 4500 & 10.2 & .004 & 7500 & 25. & .006 \\
\hline 3600 & 7.0 & .003 & 4600 & 10.6 & .004 & 8000 & 27. & .006 \\
\hline 3700 & 7.3 & .003 & 4700 & 11.0 & .004 & 8500 & 30. & .006 \\
\hline 3800 & 7.6 & .004 & 4800 & 11.4 & .004 & 9000 & 33. & .006 \\
\hline 3900 & 8.0 & .004 & 4900 & 11.8 & .004 & 9500 & 37. & .006 \\
\hline 4000 & 8.3 & .004 & 5000 & 12.3 & .004 & 10,000 & 40. & .006 \\
\hline
\end{tabular}

${ }^{\mathrm{a}} \mathrm{Eq}(81)$.

${ }^{\mathrm{b}} \mathrm{Eq}(86)$.

${ }^{\mathrm{c}} \mathrm{Eq}(89)$.

John L. Riddle read the entire manuscript and made several helpful suggestions.

\section{References}

[1] The International Practical Temperature Scale of 1968, Metrologia 5, 35 (1969).

[2] Douglas, T. B., Ball, A. F., and Ginnings, D. C., J. Research NRS 46, 334 (1951) RP2204.

[3] Bedford, R. E., Durieux, M., Muijlwijk, R., and Barber, C. R., Relationships between the International Practical Temperature Scale of 1968 and the NBS-55, NPL-61, PRMI-54, and PSU-54 temperature scales in the range from 13.81 to $90.188 \mathrm{~K}$. Metrologia 5, 47 (1969).

[4] Stimson, H. F., The International Temperature Scale of 1948, J. Research NBS 42, 209 (1949) RP1962.

[5] Temperature: Its Measurement and Control in Science and Industry, Vol. 3, ed. Herzfeld, C. M., Part 1, ed. Brickwedde, F. G. (Reinhold Publ. Corp. New York, N.Y., 1962), pp. 62-63. 